
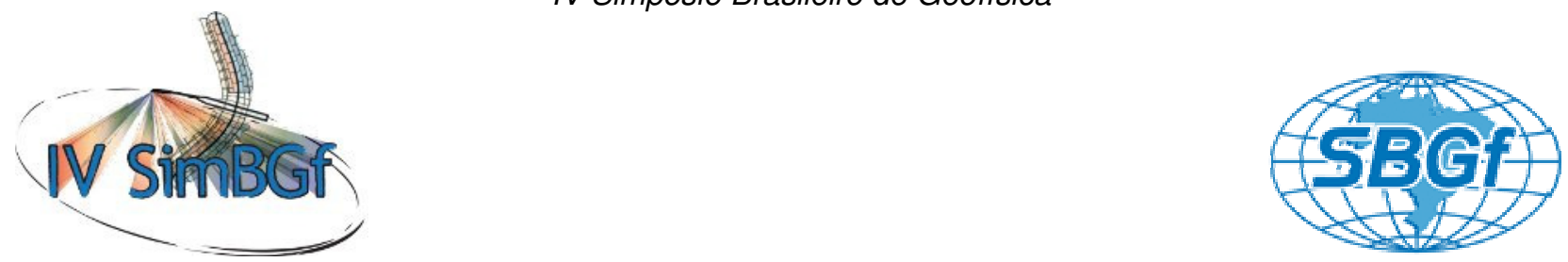

\title{
Análise Geofísica do Complexo Alcalino do Barreiro - Araxá-MG
}

Pereira, W. R., Mantovani M. S. M., Santos, R. P. Z. - Instituto de Astronomia, Geofísica e Ciências Atmosféricas - USP

Copyright 2010, SBGf - Sociedade Brasileira de Geofísica

Este texto foi preparado para a apresentação no IV Simpósio Brasileiro de Geofísica, Brasília, 14 a 17 de novembro de 2010. Seu conteúdo foi revisado pelo Comitê Técnico do IV SimBGf, mas não necessariamente representa a opinião da SBGf ou de seus associados. É proibida a reprodução total ou parcial deste material para propósitos comerciais sem prévia autorização da SBGt.

\section{Abstract}

This paper presents the results of integrated geophysical analysis of the Barreiro carbonatite-alkaline complex. The geological configuration of the complex is studied using radiometric methods and a magnetic susceptibilities and densities subsurface distribution model was compiled from magnetic and gravity data, using geophysical inversion methods.

The radionuclides concentrations measured in the surface, are typical features of alkaline-carbonatite complex, and the 3-D model generated is physically and geologically viable, showing strong correlation with the observedgeology, resulting in a maximum contrast of magnetic susceptibility of 0.235 (SI), and densities of 0.45 $\mathrm{g} / \mathrm{cm}^{3}$.

\section{Introdução}

A utilização de métodos potenciais na caracterização geofísica de intrusões alcalinas de interesse econômico constitui prática consagrada na prospecção de minerais associados a este tipo de formação, por conta da aplicabilidade de tais métodos e a resposta anômala característica de intrusivas à aplicação destes. Aliado a isto, o mapeamento superficial por gamaespectrometria apresenta contrastes nítidos de concentração de radioisótopos característicos entre domínios geológicos diferentes.

O Complexo Alcalino do Barreiro constitui um caso típico de intrusão alcalina em meio metamórfico (BIONDI, 2003), cuja associação metálica de maior interesse está na área da Mina Barreiro, de propriedade da CBMM, maior produtor mundial de Nióbio. Localizado em uma área de grande ocorrência de intrusivas alcalinas com associações minerais economicamente viáveis, o Complexo, bem como outras intrusivas locais, atrai esforços para a realização de levantamentos geofísicos em escala regional visando o mapeamento das ocorrências intrusivas locais.

A base de dados utilizada neste trabalho é constituída de levantamentos aerogeofísicos realizados por um convênio entre CPRM e CODEMIG, que reúne um conjunto amplo de dados de aerogeofísica (magnetometria e gamaespectrometria) adquiridos nos anos de 2005 e 2006, dispostos na forma de linhas paralelas N-S distantes $400 \mathrm{~m}$ compostas com linhas de controle E-W distantes 1200m, disponibilizados no formato .GDB (Geosoft Database), pré-processados e referenciados no espaço, e um levantamento gravimétrico terrestre realizado por GEOLIT/IAG/USP nos anos de 2005 e 2006, dispostos na forma de malha irregular, com densidade de dados de $500 \mathrm{~m}$ em torno da anomalia, disponibilizados em formato .GDB, reduzidos e referenciados no espaço (RUGENSKI, 2006).

\section{Metodologia/ Problema Investigado}

O Complexo Alcalino-carbonatítico do Barreiro está localizado a $10 \mathrm{~km}$ ao sul do Município de Araxá-MG, centrado no ponto $46^{\circ} 23^{\prime} \mathrm{W}$; $19^{\circ} 39^{\prime} \mathrm{S}$, e compreende uma área superficial aproximadamente circular com cerca de $4,5 \mathrm{~km}$ de diâmetro em meio a xistos e quartzitos do grupo Ibiá, na Sinforma de Araxá. O mesmo é constituído basicamente de Beforsitos e Glimeritos cuja configuração está relacionada com o evento original, iniciado pela intrusão de rocha ultrabásica, e posterior sobreposição de carbonatitos promovendo a glimeritização das ultrabásicas. Alem disso, ocorre uma forte fenitização da rocha encaixante, resultando em uma porção constituída de xistos fenitizados no entorno do Complexo (Figura 1).

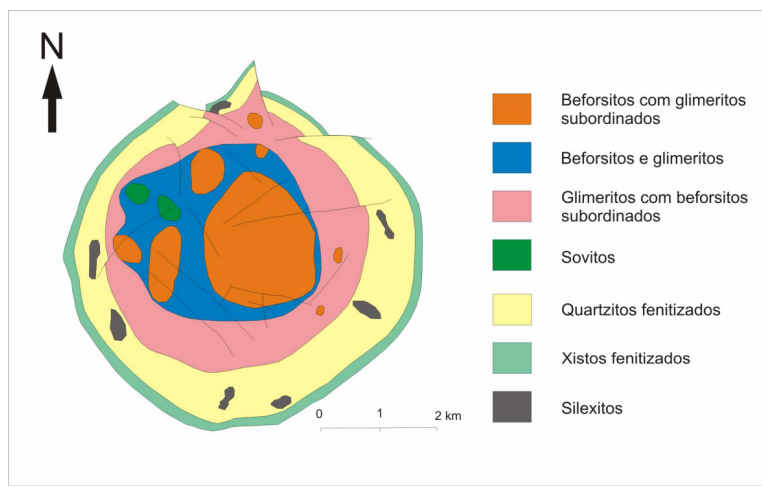

Figura 1 - Configuração Geológica do Complexo Alcalino do Barreiro. Modificado de Biondi (2003).

Procedeu-se um estudo das distribuições superficiais de radioisótopos traços ( $\mathrm{U}$, Th e $\mathrm{K}$ ), e sua relação com a composição superficial do complexo, assim como a configuração geológica regional com base em dados de Gamaespectrometria aérea de CPRM/CODEMIG.

Em seguida, para os dados de magnetometria aérea, foi removida uma superfície de tendência polinomial de ordem um, e, posteriormente, a aplicação de um filtro de continuação para cima de $400 \mathrm{~m}$, removendo assim, sinais ruidosos ou, a priori, sem significado geológico representados pelas altas freqüências.

O conjunto de dados gravimétricos e magnetométricos passaram por um processo de inversão, conforme descrito por Li e Oldenburg (1996), gerando-se um 
modelo tridimensional de distribuição de propriedades físicas em subsuperfície.

\section{Resultados}

As concentrações de radioisótopos (Figura 2-a) apresentam, na região do complexo, um alto nos valores de contagem de $U$ e Th, característicos de formações intrusivas alcalino-carbonatíticas, mostrando, alem da boa delimitação do Complexo, uma forte correlação com os domínios tectônicos da sinforma de Araxá representada na figura $2-b$.

a)

$19^{\circ} 45^{\prime}$

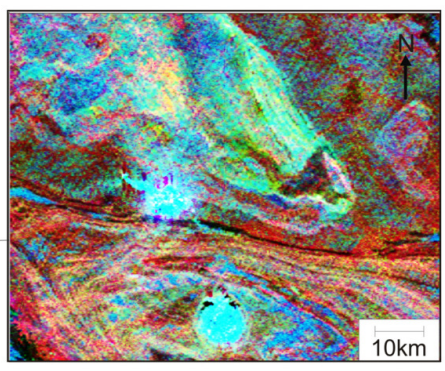

$47^{\circ} 00^{\prime}$

b)

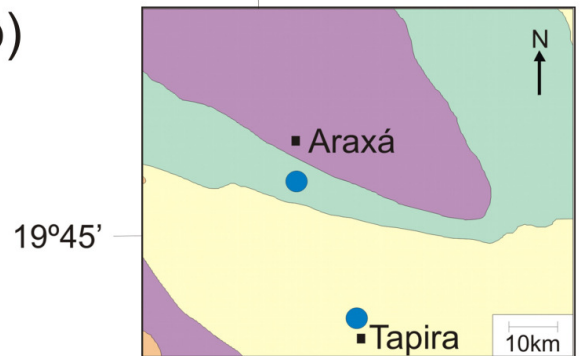

Grupo Araxá

Grupo lbiá

Grupo Canastra

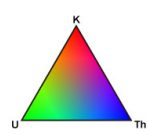

Figura 2 - Mapa de correlação entre a) contagens de elementos traços, representados no diagrama ternário $e$ b) domínios tectônicos, representados em legenda geológica, (modificado de Seer (1999).

A anomalia magnética referente ao Complexo do Barreiro (Figura 3-a) apresenta sinais localizados, de pequena extensão, e grande contraste de valores de campo total, localizados a NW e E da anomalia principal, de maior extensão representada no centro do mapa. Tal característica das anomalias acessórias, indicando tratar-se de sinais relacionados a fontes superficiais.

A anomalia magnética principal tem a característica de um dipolo típico para a esta latitude do hemisfério sul, o que indica uma magnetização remanente de tal ordem a não provocar distorções significativas no dipolo.

O mapa de anomalia Bouguer (Figura 3-b), mostra uma relação mais direta desta com o mapa da Figura 1, ou seja, um comportamento mais localizado e melhor delimitado, de formato circular, indicativo de uma distribuição radial de densidades no interior e entorno do Complexo.

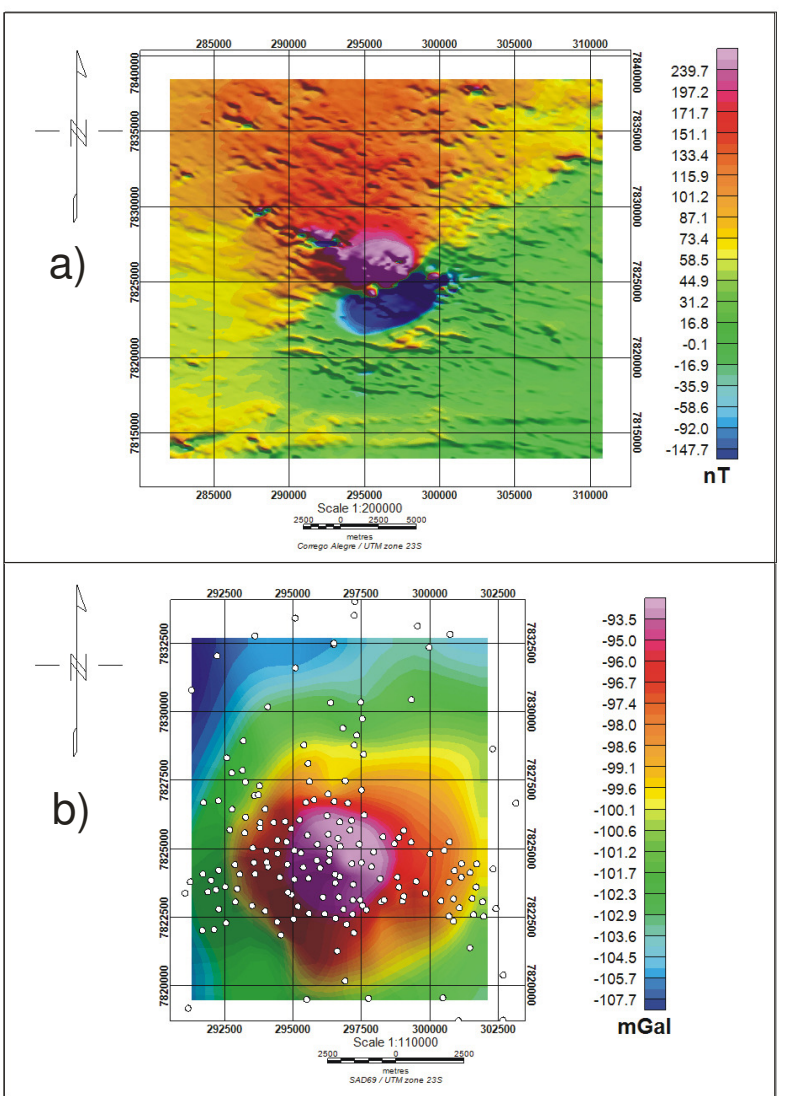

Figura 3 - a) Mapa de anomalia magnética; b) Mapa de anomalia Bouguer com densidade de pontos de aquisição .

O modelo de distribuição de suscetibilidades magnéticas, elaborado a partir dos dados de magnetometria aérea (Figura 4), apresenta distribuição irregular em sua porção superior, caracterizada por uma região de baixos valores de suscetibilidade magnética a NW do modelo, não verificado na porção média e inferior deste.

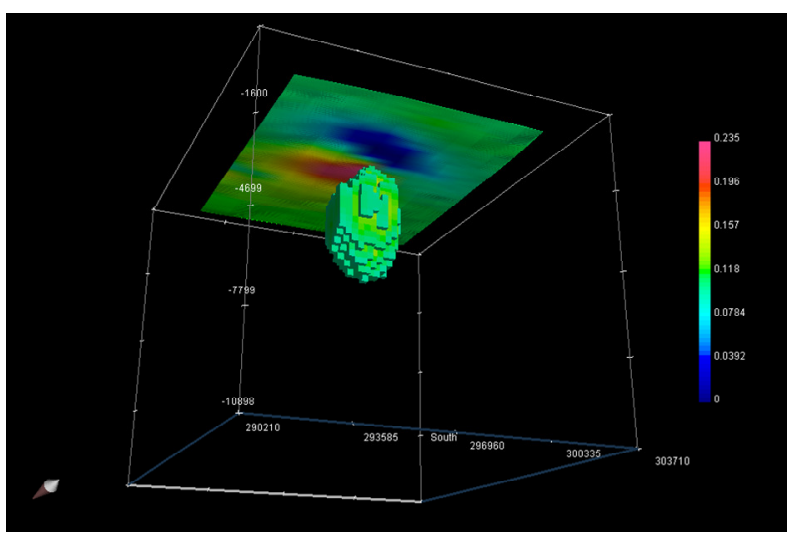

Figura 4 - Modelo de distribuição de suscetibilidade magnética em sub-superfície referente ao Complexo alcalino do Barreiro (SI). 
Já o modelo de distribuição de densidades (Figura 5), tem uma característica mais simétrica, não apresentando descontinuidades ao longo de sua superfície.

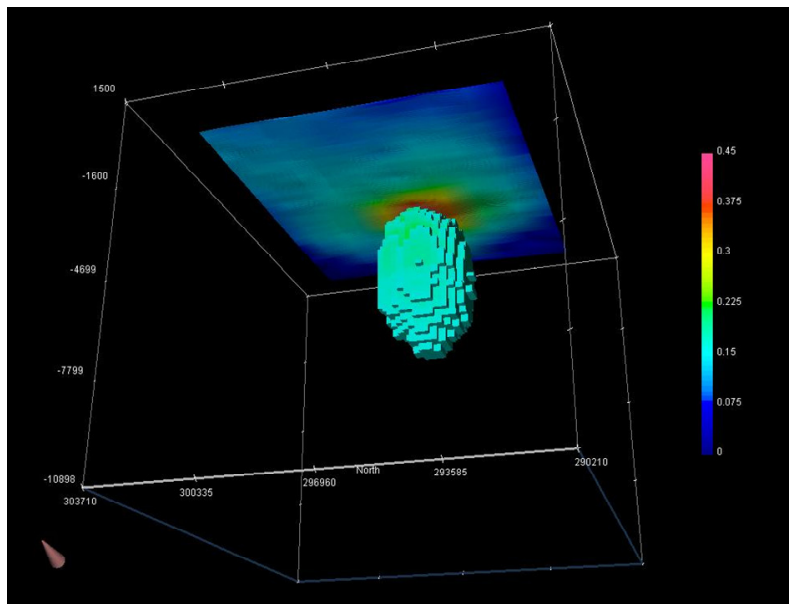

Figura 5 - Modelo de distribuição de densidade em sub-superfície referente ao Complexo alcalino do Barreiro $\left(\mathrm{g} / \mathrm{cm}^{3}\right)$.

Observando os cortes em planta de ambos os modelos, verifica-se que 0 modelo de distribuição de suscetibilidade magnética (Figura 6-a), apresenta uma tendência á concentração dos maiores valores a leste deste, no corte em planta do modelo de distribuição de densidades (Figura 6-b), o que se verifica é uma distribuição mais radial, sem deficiências significativas de concentração de valores de densidade.

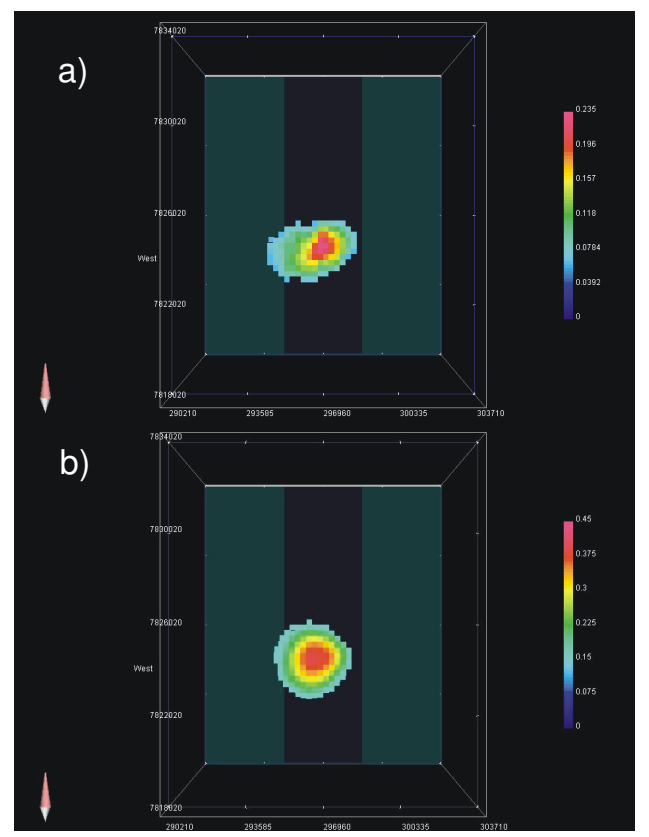

Figura 6 - a) Corte em planta do modelo de distribuição de suscetibilidade magnética (SI), e b) Corte em planta do modelo de distribuição de densidade $\left(\mathrm{g} / \mathrm{cm}^{3}\right)$, referentes ao Complexo alcalino do Barreiro.

\section{Discussão e Conclusões}

Analisando os mapas da Figura 2, é possível estabelecer uma correlação entre os domínios tectônicos que caracterizam a sinforma de Araxá, e as concentrações de $\mathrm{U}$, Th e $\mathrm{K}$, representadas em imagem ternária em sistema RGB, sendo os canais de espectrometria Gama do Urânio e Tório representados, respectivamente, pelas cores verde e azul, delimitando a alcalina, portanto, pela junção destas, resultando em uma tonalidade ciano, indicativa de um alto nas contagens de Urânio e Tório.

A Figura 3 mostra anomalias, tanto magnética como gravimétrica, bem delimitadas, sendo a anomalia Bouguer mais suave, devido à menor densidade de dados cobrindo a área estudada. Ainda assim, a anomalia magnética apresenta um dipolo característico, de acordo com o esperado para a latitude em que se encontra, sendo que, devido à maior densidade de dados, é possível verificar ocorrências de menor tamanho. Uma magnetização remanente importante requer um tratamento diferenciado dos dados para viabilizar o processo de inversão, por esse motivo, modelos sintéticos foram elaborados para estudar a influência da magnetização remanente no dipolo gerado pela alcalina, assim como simulações de dipolos por contrastes de suscetibilidades positivos para a posição, inclinação e declinação do campo, e intensidade do sinal foram elaborados. Constatou-se que um corpo sintético, com as mesmas características observadas no complexo, e sem magnetização remanente, gera dipolos com as mesmas características do gerado pelo Complexo do Barreiro, o que é indicativo de uma magnetização remanente pouco importante.

Analisando os modelos das Figuras 4 e 5, assim como as distribuições internas de propriedade física observadas na Figura 6, é possível inferir que a distribuição de suscetibilidade magnética está relacionada com a composição do Complexo (Figura 1), onde, devido à ocorrência de Sovitos na porção NW do complexo, existe uma região de baixos valores de suscetibilidade com relação à porção centro-leste, onde há maior ocorrência de Beforsitos. As densidades se distribuem radialmente de maneira uniforme, indicando estarem relacionadas à configuração espacial do complexo, e correspondem a um volume calculado de $37 \mathrm{~km}^{3}$ para o limite mínimo de densidades de $0,15 \mathrm{~g} / \mathrm{cm}^{3}$, e máximo de $0,45 \mathrm{~g} / \mathrm{cm}^{3}$.

Um modelo tridimensional único pode ser elaborado a partir da sobreposição das duas propriedades, dando uma idéia de como tais propriedades se relacionam no espaço, conforme representado na Figura 7.

O processo de inversão apresenta resultados viáveis do ponto de vista geofísico, uma vez que as dimensões do modelo são da mesma ordem do observado, com 4,5km de diâmetro no maior eixo (BIONDI, 2003), alem de apresentar uma diferença de diâmetro entre o modelo de densidades em relação ao de suscetibilidade magnética, - que é razoável considerando que a diferença de densidades se estende ao entorno devido à natureza do processo intrusivo, enquanto os contrastes de suscetibilidade magnética estão relacionados à composição interna do Complexo, e à diferença desta com relação ao entorno. 


\section{Referências}

BIONDI, J. C., Processos metelogenéticos e os depósitos minerais brasileiros. São Paulo: Oficina de Textos, 2003, 78-79.

BHATTACHARYYA, B. K. Magnetic anomalies due to prism-shaped bodies with arbitrary magnetization: Geophysics, 29, 517-531, 1964.

CARMICHAEL, R. S. Handbook of physical properties of rocks. CRC Press, 1982.

CPRM/CODEMIG. Relatório final do levantamento e processamento dos dados magnetométricos e gamaespectrométricos, área7, Patos de Minas, Araxá, Divinópolis. [Belo Horizonte], CPRM/CODEMIG, 2006.

DUTRA, A. C. Estudo de anomalia gravimétricas e aeromagnéticas das alcalinas de Morro do Engenho e A2, sudoeste de Goiás: 2006. 73f. Dissertação (Mestrado em Geofísica) - Instituto de Astronomia, Geofísica e Ciências Atmosféricas, Universidade de São Paulo, São Paulo, 2006.

FILHO, A. I., LIMA, P. R. A.; SOUZA, O. M., Aspects of geology of the Barreiro Carbonatitic Complex, AraxáMG, Brazil. São Paulo: CBMM, 1984.

GRAV3D; A Program Library for Forward Modelling and Inversion of Gravimetric Data over 3D Structures, version 4.0: Developed under the consortium research project Joint/Cooperative Inversion of Geophysical and Geological Data, UBC-Geophysical Inversion Facility, Department of Earth and Ocean Sciences, University of British Columbia, Vancouver, UBC, 2006.

LI, Y.; OLDENBURG, D. W. 3-D inversion of magnetic data, Geophysics, 1996, 61, 394-408.

LI, Y.; OLDENBURG, D. W. 3-D inversion of gravity data, Geophysics, 63, 109-119, 1998.

MAG3D; A Program Library for Forward Modelling and Inversion of Magnetic Data over 3D Structures, version 4.0: Developed under the consortium research project Joint/Cooperative Inversion of Geophysical and Geological Data, UBC-Geophysical Inversion Facility,
Department of Earth and Ocean Sciences, University of British Columbia. Vancouver: UBC, 2006.

MORAES, L. C.; SEER. H. J.; BENTO J. H. B.; LEAL. C. F.; MADEIRA. M. R. Análise geométrica do alojamento de magmas no entorno do Complexo AlcalinoCarbonatítico do Barreiro, Araxá, Minas Gerais, por meio da atitude espacial de fraturas e diques. In: IV SIMPÓSIO DE VULCANISMO E AMBIENTES ASSOCIADOS. 2008, Foz do Iguaçu. Anais do IV Simpósio de Vulcanismo e Ambientes Associados. Foz do Iguaçu: SBG, 2008.

RUGENSKI. A. Investigação geofísica dos complexos alcalinos do sul e sudeste do Brasil. 2006. 352f. Dissertação (Doutorado em Geofísica) - Instituto de Astronomia, Geofísica e Ciências Atmosféricas, Universidade de São Paulo, São Paulo, 2006.

SEER, H., 1999, Evolução tectônica dos grupos Araxá e lbiá na sinforma de Araxá-MG. 1999. Dissertação (Doutorado em Geologia) - Instituto de Geociências, Universidade de Brasília, Brasília, 1999.

SHARMA, P.V., Rapid computation of magnetic anomalies and demagnetization effects caused by bodies of arbitrary shape: Pure Appl. Geophys., 1966, 64, 89-109.

TOULOUKIAN, Y. S., JUDD, W. R., ROY, R. F. Physical properties of rocks and minerals, McGraw-Hill, 1981.

TELFORD, W. M., GELDART L.P., SHERIFF R. E., KEYS D. A, Applied Geophysics, Cambridge: University Press. 1998. 


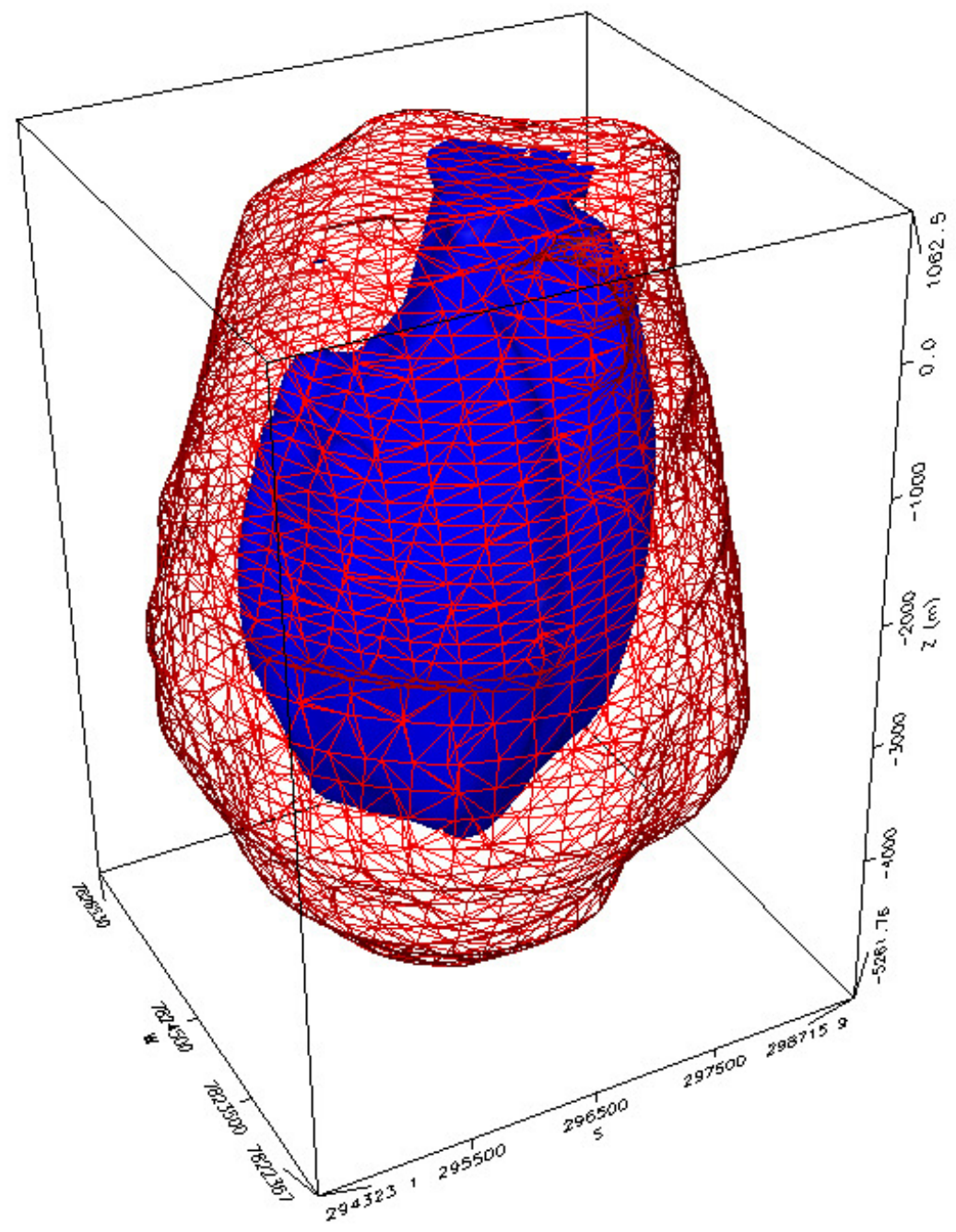

Figura 7 - Modelo tridimensional de distribuição de propriedades físicas do Complexo Alcalino do Barreiro: Suscetibilidade magnética (superfície azul) e densidade (grade vermelha). 